\title{
VIEWS OF THE PROCESS AND CONTENT OF ETHICAL REVIEWS OF HIV VACCINE TRIALS AMONG MEMBERS OF US INSTITUTIONAL REVIEW BOARDS AND SOUTH AFRICAN RESEARCH ETHICS COMMITTEES
}

ROBERT KLITZMAN

\section{Keywords}

international bioethics, ethical relativism, decision-making, developing world, gender,

race, institutional contexts, informed consent.

\section{ABSTRACT}

Given the ethical controversies concerning HIV vaccine trials (HVTs), we aimed to understand through an exploratory study how members of institutional review boards (IRBs) in the United States (US) and research ethics committees (RECs) in South Africa (SA) view issues concerning the process and content of reviews of these studies. We mailed packets of 20 questionnaires to 12 US IRB chairs and administrators and seven REC chairs to distribute to their members. We received 113 questionnaires (76 from the US and 37 from SA). In both countries, members tended to be white males with advanced academic degrees. Compared to the US, SA members called for 'major changes' in HVT protocols more frequently $(p=0.004)$, and were less likely to think that HVT participants understood risks and benefits $(p=0.033)$ or informed consent forms $(p=0.000)$. In both countries, members were divided on several critical issues (e.g. the minimum standard for treatment for HVT participants who became infected during the HVT), but agreed that they needed more training. Of the SA respondents, $40 \%$ reported that they were 'self-taught' in ethics. This study, the first we know of to offer quantitative data comparing US vs. non-US IRBs/RECs, thus suggests key similarities and differences (e.g. compared to SA respondents, US respondents appeared to overestimate participants' understanding of informed consent), along with needs for education. These initial exploratory data in this area have important implications for IRBs, RECs, policy-makers and scholars concerning future practice, training, policy, and investigations in research ethics, and prevention and treatment of HIV and other diseases in the developing world and elsewhere.

Address for correspondence: Robert Klitzman, MD, Associate Professor of Clinical Psychiatry, Columbia University College of Physicians and Surgeons, 1051 Riverside Drive, Unit 15, New York, NY 10032, USA. rlk2@columbia.edu 
The development of safe and effective preventive HIV vaccines has been seen as critical for stemming the growing global HIV pandemic, ${ }^{1}$ yet HIV vaccine trials (HVTs) have posed difficult ethical dilemmas. ${ }^{2}$ Little empirical research has been done on how key stakeholders - for example, policy-makers, clinicians, members of institutional review boards (IRBs) or research ethics committees (RECs) - view and approach these issues. Yet, such data could be vital in these areas, helping to move HVTs forward. Debates about HVTs succeed a long series of ethical challenges and controversies about HIV since the onset of the epidemic. ${ }^{3}$ Though HIV medications are becoming more available in the developing world, they help only those already infected; access to medications, though widening, remains limited (e.g. increasing from about $1 \%$ to $17 \%$ of patients in need in sub-Saharan Africa between 2002 and 2005). ${ }^{4}$ Historically, vaccines, in general, have also raised complex ethical quandaries, causing intense debates in the United States (US) and abroad. ${ }^{5}$

On several key ethical issues concerning HVTs, the Joint United Nations Programme on HIV/AIDS (UNAIDS) failed to reach consensus, and ultimately referred these disagreements to local IRBs/ RECs, which thus now serve as 'final arbiters' in

${ }^{1}$ B. Bloom. The Highest Attainable Standard: Ethical Issues in AIDS Vaccines. Science 1998; 279: 186-188; Republic of South Africa: Department of Health. 2002. Summary Report: National HIV and Syphilis Antenatal Sero-prevalence Survey in South Africa 2002. Pretoria: Department of Health. Available at: www.doh.gov.za/docs/reports/ 2002/hiv-syphilis.pdf [Accessed 26 Dec 2006].

${ }^{2}$ M. Angell. Ethical Imperialism? Ethics in International Collaborative Clinical Research. N Engl J Med 1988; 319: 1081-1083; P. Lurie \& S. Wolfe. Unethical Trials of Interventions to Reduce Perinatal Transmission of the Human Immunodeficiency Virus in Developing Countries. $N$ Engl J Med 1997; 337: 853-856.

${ }^{3}$ R. Bayer. 1991. Private Acts, Social Consequences: AIDS and the Politics of Public Health. New Brunswick, NJ: Rutgers University Press; R. Klitzman \& R. Bayer. 2003. Mortal Secrets: Truth and Lies in the Age of AIDS. Baltimore, MD: Johns Hopkins University Press.

${ }^{4}$ United Nations Programme on HIV/AIDS (UNAIDS). 2003. Accelerating Action Against AIDS in Africa. Geneva: UNAIDS. Available at: data.unaids.org/UNA-docs/ICASA_Report_2003_en.pdf [Accessed 6 Dec 2006]; United Nations Programme on HIV/AIDS (UNAIDS). 2006. Global Access to HIV Therapy Tripled in Past Two Years, but Significant Challenges Remain. Geneva: UNAIDS. Press release, 28 March. Available at: http://www.data.unaids.org/pub/PressRelease/ 2006/20060328-PR-3by5_en.pdf?preview=true [Accessed 6 Dec 2006].

${ }^{5}$ United Nations Programme on HIV/AIDS, 2003, ibid; United Nations Programme on HIV/AIDS, 2006, ibid. determining whether and how HVTs are conducted in particular communities. ${ }^{6}$ Yet, how local IRBs/ RECs view and face these dilemmas generated by HVTs remains unknown. Some critics consider UNAIDS' guidance document too 'aspirational' and not sufficiently 'pragmatic'. ${ }^{7}$ Local IRBs/RECs now face several major unresolved controversies concerning HVTs. For example, ethicists have intensely debated the minimum standard of treatment for HVT participants who become HIVinfected while participating in the trial, with some critics arguing that the standard should be the highest available in the sponsoring country rather than the highest available or standard level of treatment in the host country, ${ }^{8}$ while others argue that such a standard may not be 'attainable and sustainable'. ${ }^{9}$ Yet reports about IRB decision-making concerning HVTs have largely been theoretical. ${ }^{10}$ Thus, crucial questions remain as to how IRBs/RECs view and approach these and related issues concerning HVTs, and differ in doing so. These questions are important because IRBs/RECs may potentially benefit from assistance or guidance in clarifying and/or weighing these issues. Yet, how these dilemmas are perceived or handled within IRBs/RECs remains unknown.

IRBs in the US, and RECs, as they are called in many other countries, are increasing in number and importance, reviewing HVTs and other international research. But, overall, little empirical research has been done on IRB/REC members' views of ethical issues. A study of 37 REC chairs or administrators from several African countries indicated

\footnotetext{
${ }^{6}$ R. Bayer. Ethical Challenges of HIV Vaccine Trials in Less Developed Nations: Conflict and Consensus in the International Arena. AIDS 2000; 14: 1051-1057.

${ }^{7}$ R. Macklin. Four Forward-looking Guidance Points. Developing World Bioeth 2001; 1: 121-134.

${ }^{8}$ M. Angell. The Ethics of Clinical Research in the Third World. $N$ Engl J Med 1997; 37: 847-849.

${ }^{9}$ R.J. Levine. Some Recent Developments in the International Guidelines on the Ethics of Research Involving Human Subjects. Ann $N Y$ Acad Sci 2000; 918: 170-178.

${ }^{10}$ B. Haynes. Scientific and Social Issues of Human Immunodeficiency Virus Vaccine Development. Science 1993; 260: 1279-1286; J. Feinberg \& A. Japour. Scientific and Ethical Considerations in Trial Design for Investigational Agent for the Treatment of Human Immunodeficiency Virus Infection. Clin Infect Dis 2003; 36: 201-206; C. Grady. 1995. The Search for an AIDS Vaccine: Ethical Issues in the Development and Testing of a Preventative HIV Vaccine. Bloomington, IN: Indiana University Press; Macklin, op. cit. note 7 .
} 
that they thought their capacity to review HVTs was 'moderate to limited' and that they had several training needs concerning the science and ethics of HVTs. ${ }^{11}$ Yet we know of no published study comparing IRBs in the US versus RECs in the developing world in any way; and numerous critical questions remain as to whether members or boards and committees in any one country or in different countries vary in their views and approaches toward ethical issues and, if so, how. These questions are vital because IRBs/RECs have been facing criticism and numerous complex issues. Specifically, discrepancies have been found to exist among IRBs, including inconsistencies in their deliberations, approvals ${ }^{12}$ and applications of ethical standards. ${ }^{13}$ In addition, ethical relativism has been intensely debated. Some argue that bioethics should be universalistic and not vary between cultures, ${ }^{14}$ that a 'common morality' exists, ${ }^{15}$ and that embracing 'ethical relativism' precludes the ability to criticize another's ethical position. ${ }^{16}$ But others argue that 'ethics are local' not universal, ${ }^{17}$ that 'multiple moral traditions' exist even in the US, that ethical principles should be amended in different cultural contexts, and that a 'common morality' does not exist. ${ }^{18}$ Tensions

11 C. Milford, D. Wassenaar \& C. Slack. Resources and Needs of Research Ethics Committees in Africa: Preparations for HIV Vaccine Trials. IRB 2006; 28(2): 1-9.

12 J. Bell, J. Whiton \& S.Connelly. James Bell Associates. 1998. Evaluation of NIH Implementation of Section 491 of the Public Health Service Act, Mandating a Program of Protection for Research Subjects. Report prepared under a National Institute of Health contract, N01-OD-22109. Washington, DC: United States Department of Health and Human Services.

13 J. Goldman \& M. Katz. Inconsistency and Institutional Review Boards. JAMA 1982; 248: 197-202; A. Doob. The Reliability of Ethical Reviews: Is it Desirable? Can Psychol 1983; 24: 269-270; W. Eaton. The Reliability of Ethical Reviews: Some Initial Empirical Findings. Can Psychol 1983; 24: 14-18.

14 Angell, op. cit. note 2; Lurie \& Wolfe, op. cit. note 2.

15 T.L. Beauchamp. A Defense of the Common Morality. Kennedy Inst Ethics $J$ 2003; 13: 259-274.

${ }^{16}$ R. Macklin. 1991. Against Relativism, Cultural Diversity and the Search for Ethical Universals in Medicine. New York, NY: Oxford University Press.

${ }^{17}$ N. Christakis. Ethics are Local: Engaging Cross Cultural Variation in the Ethics for Clinical Research. Soc Sci Med 1992; 35: 1079-1091.

18 L. Turner. Zones of Consensus and Zones of Conflict: Questioning the 'Common Morality' Presumption in Bioethics. Kennedy Inst Ethics $J$ 2003; 13: 193-218; M. Barry. Ethical Consideration of Human Investigation in Developing Countries: The AIDS Dilemma. $N$ Engl J Med 1992; 319: 1083-1086. between universal versus local contexts date from the founding of IRBs, which were established to be 'local', making decisions for an institution, ${ }^{19}$ though the principles underlying deliberations were to be universal. Given discrepancies, some critics call for national rather than local review of protocols, ${ }^{20}$ though others disagree. ${ }^{21}$ Ethicists have debated this question in the literature, ${ }^{22}$ but we have found no prior data on how IRB/REC members themselves view this question. IRB members' goals to be impartial have also been criticized as potentially being elusive, given members' demographics, since most are white and male and work at research institutions, and thus may be 'biased' in favor of research. $^{23}$

In the developing world, these difficulties may be compounded, as specific challenges emerge in importing, establishing, and incorporating the idea of IRBs into other cultures. For example, in one of the only studies of researchers in the developing world, 29\% said most of their research collaborators in foreign countries did not speak or read English well. ${ }^{24}$ Principal investigators (PIs) and IRB/REC members in eight countries saw the US IRB review process as 'unnecessarily burdensome' in time and difficulty, ${ }^{25}$ and perceived little flexibility in how US regulations were applied, despite cultural differences. Moreover, in other cultures, illness itself is

\footnotetext{
${ }^{19}$ See United States Department of Health and Human Services (HHS). 2005. Code of Federal Regulations. Title 45. Part 46. Protection of Human Research Subjects. Washington, DC: HHS. Available at: http://www.hhs.gov/ohrp/humansubjects/guidance/45cfr46.htm [Accessed 23 Dec 2006].

${ }^{20}$ L. Eckenwiler. Moral Reasoning and the Review of Research Involving Human Subjects. Kennedy Inst Ethics J 2001; 11: 37-69; M. Christian et al. A Central Institutional Review Board for Multi-institutional Trials. N Engl J Med 2002; 346: 1405-1408.

${ }^{21}$ D. Phillips. Institutional Review Boards Under Stress: Will they Explode or Change? JAMA 1996; 276: 1623-1626.

${ }^{22}$ Ibid; Eckenwiler, op. cit. note 20; Christian et al., op. cit. note 20.

${ }^{23}$ Grady, op. cit. note 10 .

${ }^{24}$ A. Wichman et al. Collaborative Research Involving Human Subjects: A Survey of Researchers Using International Single Project Assurances. IRB 1997; 19: 1-6.

${ }^{25}$ J. Sugarman et al. 2001. International Perspectives on Protecting Human Research Subjects. In Ethical and Policy Issues in International Research: Clinical Trials in Developing Countries: Vol. II. National Bioethics Advisory Commission (NBAC). Bethesda, MA: NBAC: E1-E30. Available at: http://www.georgetown.edu/research/nrcbl/nbac/ clinical/Vol2.pdf [Accessed 6 Dec 2006].
} 
often perceived and treated in fundamentally different ways. ${ }^{26}$

Though seriously under-studied, IRBs in the US and RECs in the developing world are growing rapidly, with 3,000-5,000 IRBs now in the US, ${ }^{27}$ ranging from 5-44 members. ${ }^{28}$ The mean numbers of members for high and low volume IRBs are 20 and 10 , respectively. ${ }^{29}$

South Africa (SA) serves as an important country in which to explore these issues as a case in point because its HIV prevalence is among the highest in the world and HVTs are being conducted there. SA has approximately 23 RECs. ${ }^{30}$ Of these, 12 are registered and have assurances with the US Office of Human Research Protections (OHRP). ${ }^{31}$

Thus, several critical research questions emerge: How do members of US IRBs and SA RECs view and approach these vital issues concerning HVTs? Controversies about HVTs take place in the context of, and may reflect, broader debates about IRBs more generally (e.g. concerning participant understanding of consent forms, central versus local

${ }^{26}$ R. Fox. 1959. Experiment Perilous. Glencoe: The Free Press; R. Fox \& J. Swazey. 1992. Spare Parts: Organ Replacement in American Society. New York, NY: Oxford University Press; B. Malinowski. 1950. Argonauts of the Western Pacific: An Account of Native Enterprise and Adventure in the Archipelagoes of Melanesian New Guinea. New York, NY: E.P. Dutton; E.E. Evans-Pritchard. 1937. Witchcraft, Oracles, and Magic Among the Azande of the Anglo-Egyptian Sudan. Oxford: Clarendon Press; J. Camaroff \& J. Camaroff. Occult Economies and the Violence of Abstraction: Note from the South African Postcolony. American Ethnologis 1999; 26: 279-303.

${ }^{27}$ Office of Inspector General. 1998. Final Report on Low Volume Institutional Review Boards. DHHS Publication No. OEI-01-97-00194. Washington, DC: United States Government Printing Office. Available at: http://www.oig.hhs.gov/oei/reports/oei-01-97-00194.pdf [Accessed 6 April 2006].

28 A. Anderson. 2002. RIID Human Subjects Protection Program Best Practices. Human Subjects Research Review Board Meeting. Frederick: MD. Seminar presentation, 5 September. Available at: http:// www.geocities.com/artnscience/AO_Anderson_CV/index.html

[Accessed 6 Apr 2006]; United States Department of Health and Human Services, op. cit. note 19.

${ }^{29}$ Bell et al., op. cit. note 12.

${ }^{30}$ World Health Organization (WHO)/United Nations Programme on HIV/AIDS (UNAIDS). African AIDS Vaccine Program (AAVP). 2003. African AIDS Vaccine Program: Inventory. Online: AAVP. Available at: http://www.saavi.org.za/inventory.htm [Accessed 16 Dec 2006].

${ }^{31}$ See Office of Human Research Protections (OHRP). International Assurances and Components South Africa (106). Available at: http:// www.ohrp.cit.nih.gov/search/asurlst.asp?locationid=228\&atype $=$ f\&otype $=$ i\&DLST $=$ Submit\&ctype $=\mathrm{i} \&$ key $1=$ F\&key $2=$ M\&choice $=$ b\&name $=$ AFGHANISTAN [Accessed 16 Jan 2007]. review, ethical relativism, PI wariness of IRBs, etc.). These areas, such as whether study participants understand informed consent forms, are important to ensure adequate and appropriate protections of human subjects as articulated in the Belmont Report $^{32}$ and the Declaration of Helsinki. ${ }^{33}$ Hence, debates about reviews of HVTs concern not just the content of HVTs, but the process of the IRB review involved, and these areas are consequently critical to probe. Do US IRBs and SA RECs differ in their views of process or content of HVT reviews and, if so, how? Given the lack of available data, and the controversies involved, this study aimed to shed initial light on whether and how members of US IRBs and SA RECs may differ with regard to three broad areas: 1) content of HVT reviews, 2) process of HVT reviews, and 3) process and functioning of IRB/REC reviews in general. Specifically, as an initial exploratory study in this area, this research probed several key questions: whether IRBs and RECs differ in content variables (e.g. perceptions of HVT participants' understandings of risks and benefits, placebos and informed consent, approaches toward ethical debates concerning standards of treatment for HVT participants who become infected during the HVT, and decisions about oral consent in HVTs); HVT-related process issues (e.g. requests for major changes in HVT protocols, and IRB/REC disagreements about HVTs); and general process issues (e.g. types and background of members, communication with IRBs/RECs in other countries, needs for central versus local review, amendment of ethical principles in other countries, IRB education needs, and views of PI understandings of IRBs). Because little data in these areas are available, the overall goals were to gain insights that might help further education, research and guidelines.

\footnotetext{
32 The National Commission for the Protection of Human Subjects of Biomedical and Behavioral Research. 1979. The Belmont Report: Ethical Principals and Guidelines for the Protection of Human Subjects of Research. Washington, DC: Department of Health, Education, and Welfare, National Commission for the Protection of Human Subjects of Biomedical and Behavioral Research. Available at: http://www.usd.edu/ oorsch/compliance/SOPs/APPENDIXA.pdf [Accessed 23 Dec 2006].

${ }^{33}$ World Medical Association (WMA). 1964. Declaration of Helsinki. As amended by the WMA $52^{\text {nd }}$ General Assembly, Hong Kong, 1989. Ferney-Voltaire: WMA. Availaible at: http://www.onlineethics.org/ reseth/helsinki.html [Accessed 23 Dec 2006].
} 


\section{METHODS}

By researching the scientific literature and contacting the HVT Network (HVTN), the HIV Prevention Trials Network (HPTN), the International AIDS Vaccine Initiative (IAVI) and the National Institutes of Health (NIH), we compiled a list of 42 US institutions that have conducted HVTs in the US and/or overseas. ${ }^{34} \mathrm{We}$ crosschecked this list with the database of IRBs with OHRP assurances (dated Nov 2003). We found that some information on the OHRP database was outdated (e.g. incorrect contact information) and not all institutions on our list were on the OHRP database. The database had accurate contact information for 27 IRB representatives, who we then contacted. We received 12 agreements to participate and only one IRB chair declined to participate, due to its policy that it does not participate in studies. Our initial research in 2004 indicated that three RECs in SA had reviewed HVTs. Due to this fact, coupled with the high prevalence of HIV in SA, we compiled a list of all 12 RECs in SA that have assurances from OHRP, including those that have undertaken HVTs. We contacted three South African medical ethicists involved in HVTs who suggested the names of seven institutions that they felt were also likely to have conducted HIV research. We contacted these RECs and received seven affirmative replies. We developed a questionnaire based on a review of the literature and interviews and discussions with members of 12 US IRBs and 10 developing world RECs. As discussed above, we designed questions to understand how IRB/REC members viewed relevant process and content issues concerning reviews of HVTs. We pilot tested the questionnaire with five respondents, and then refined the instrument. We sent questionnaires to chairs, first in the US and then in SA. Based in part on additional conversations with IRB

\footnotetext{
${ }^{34}$ See HIV Vaccine Trials Network (HVTN). 2004. HIV Vaccine Trials Network. Online: HVTN. Available at: http://www.hvtn.org [Accessed 24 Jan 2004]; International AIDS Vaccine Initiative (IAVI). 2003. International AIDS Vaccine Initiative. Online: IAVI. Available at: http:// www.iavi.org [Accessed 30 Oct 2003]; HIV Prevention Trials Network (HPTN). 2006. HIV Prevention Trials Network. Online: HPTN. Available at: http://www.hptn.org/index.htm [Accessed 23 Jan 2007]; Information obtained from HIVNet. Available at: http://www.ahrq.gov/ data/hivnet.htm [Accessed 6 Dec 2006]; Bell et al., op. cit. note 12.
}

members in the interim, we then added several questions to the instrument mailed to SA. (Instruments are available upon request from the author.)

We sent packets of 20 questionnaires to each chair. Prior work showed that mean numbers of members of high and low volume IRBs are 19.7 and 10.5 , respectively. ${ }^{35}$ IRB chairs were able to make additional copies or to inform us that they would like additional questionnaires sent to them, if needed. We mailed these questionnaires twice to each of the 12 US and 7 SA representatives who had agreed to participate and distribute these questionnaires to their members. Past attempts to study IRBs have been limited because lists of IRB/REC members are not public knowledge, and in other prior studies, chairs have often refused to provide these names. ${ }^{36}$ Moreover, for an IRB to be observed, all members must provide informed consent, which has posed obstacles in terms of feasibility. These obstacles explain in part why so few studies of IRB/ REC members have been published to date. ${ }^{37}$

In the present study, the questionnaire did not ask respondents to provide their name or the names of their institution or IRB/REC, as the questionnaire addressed controversial and potentially sensitive areas about decisions and behaviors of respondents and their IRB/RECs. Hence, potential respondents might avoid completing or returning the survey because of concerns that they or their board or institution might potentially be identified. Anonymity was thought to be important to encourage participation of potential subjects. Potential participants were also busy professionals. Thus, to reduce the potential burden to them in terms of time and effort, the questionnaire was made as short as possible (i.e. to fit on one piece of paper), though that limited the number of items that could be included. For analyses, we performed simple descriptive statistics and chi-squares to compare respondents

\footnotetext{
${ }^{35}$ T.H. Wagner et al. 2001. Technical Appendix for the Cost of Operating Institutional Review Boards (IRBS). Presented at Human Subjects Research Subcommittee - Committee on Science, National Science and Technology Council, Washington, DC: January 10.

${ }^{36}$ R. De Vries \& C. Forsberg. What do IRBs Look Like? What Kind of Support do they Receive? Account Res 2002; 9: 199-216; R. De Vries \& D.A. DeBruin. Ethics Review of Social, Behavioral, and Economic Research: Where Should we go from Here? Ethics Behav 2004; 14: 351-368.

${ }^{37}$ De Vries \& Forsberg, ibid.
} 
from the two countries. IRB approval was obtained from the author's institution.

\section{RESULTS}

We received a total of 113 completed questionnaires - including 76 from US IRB members and 37 from SA REC members, suggesting response rates of approximately 76/240, (31.7\%), and 37/140 (26.4\%), respectively. Tables 1 and 2 summarize demographics and responses of the sample. In brief, in both the US and SA, gender differences arose among respondents in highest level of education obtained $(\mathrm{p}=0.003)$; and in the US, males were also more likely to be white $(\mathrm{p}=0.012)$, and chairs $(\mathrm{p}=0.013)$.

Of the US respondents, 52 had reviewed HVTs in the US and 30 had reviewed HVTs in the developing world. Though all of these US IRBs had in fact at one point reviewed HVTs, not all current members reported having done so, or knowing that their IRB had done so. As indicated in the tables, for the purpose of analyses, for questions concerning HVTs in general and in the developing world, we included only those subjects who had in fact themselves reviewed a protocol from each of these two categories, respectively.

Concerning HVTs, of US respondents, most thought participants in the developing world understood that they might be given a placebo and understood risks and benefits of HVTs 'very' or 'somewhat well' (vs. 'poorly' or 'somewhat poorly'). Only $10.6 \%$ of these respondents had ever approved oral consent for these studies. The sample was divided on issues of standard of treatment for HVT participants who become HIV-infected during the HVT. Concerning IRB processes in general, twothirds thought their IRB would benefit from additional education. Respondents were divided on whether protocols should be reviewed centrally rather than locally and how often ethical principles should be 'amended' by IRBs/RECs in the developing world. Almost one-half (47.9\%) of US respondents thought their IRB communicates with foreign IRBs 'rarely' or 'never'.

Most SA respondents thought HVT participants understood the risks and benefits involved 'somewhat poorly' and that study participants in their country understood consent forms 'somewhat poorly', and $42.3 \%$ felt participants understood that they might be given a placebo 'poorly' or 'somewhat poorly'. On the minimum standard of treatment provided to participants of SA HVTs who become HIV infected, respondents were divided: 63.0\% believed it should be the best available worldwide, $11.1 \%$ the best available in South Africa, and 25.9\% the standard treatment in SA. Only 7.4\% had ever approved oral consent for HVTs; $75.8 \%$ believed that they would benefit from additional education regarding HVTs.

Concerning IRB processes in general, SA respondents were divided as to whether protocols should be reviewed at a centralized national level rather than locally; and $70 \%$ felt that the ethical principles used by US IRBs need to be amended by RECs 'somewhat' or 'very much'.

As shown in Table 3, on questions concerning ethics training, posed to SA REC but not US IRB members, $40 \%$ of SA respondents described themselves as 'self-taught', with males more likely than women to do so $(\mathrm{p}=0.006)$.

Comparisons of US versus SA responses revealed both similarities and differences. SA respondents had, on average, a higher level of education ( $p=0.043$ ), with over $70.2 \%$ holding $\mathrm{PhD}$ or MD degrees (vs. $38.2 \%$ of US respondents). Respondents in SA reported calling for 'major changes' in HVT protocols $(\mathrm{p}=0.004)$, more than did those in the US. Compared to US respondents, SA members thought that HVT participants understood risks and benefits less $(\mathrm{p}=0.033)$ and that participants in general understood informed consent forms less well $(p=0.000)$. In both countries, respondents agreed that their IRBs rarely approved oral consent for an HVT and sometimes disagreed about HVTs. In both countries, most respondents did not feel that communication with foreign IRBs was adequate and members were divided on the minimal acceptable standard of treatment for HVT participants who became HIV-infected during the HVT. Respondents also felt that researchers understood the role of the IRBs/RECs only somewhat well, that ethical principles needed to be amended somewhat in the developing world, and that members needed more education. Among US and SA respondents combined, there were no gender differences in views 


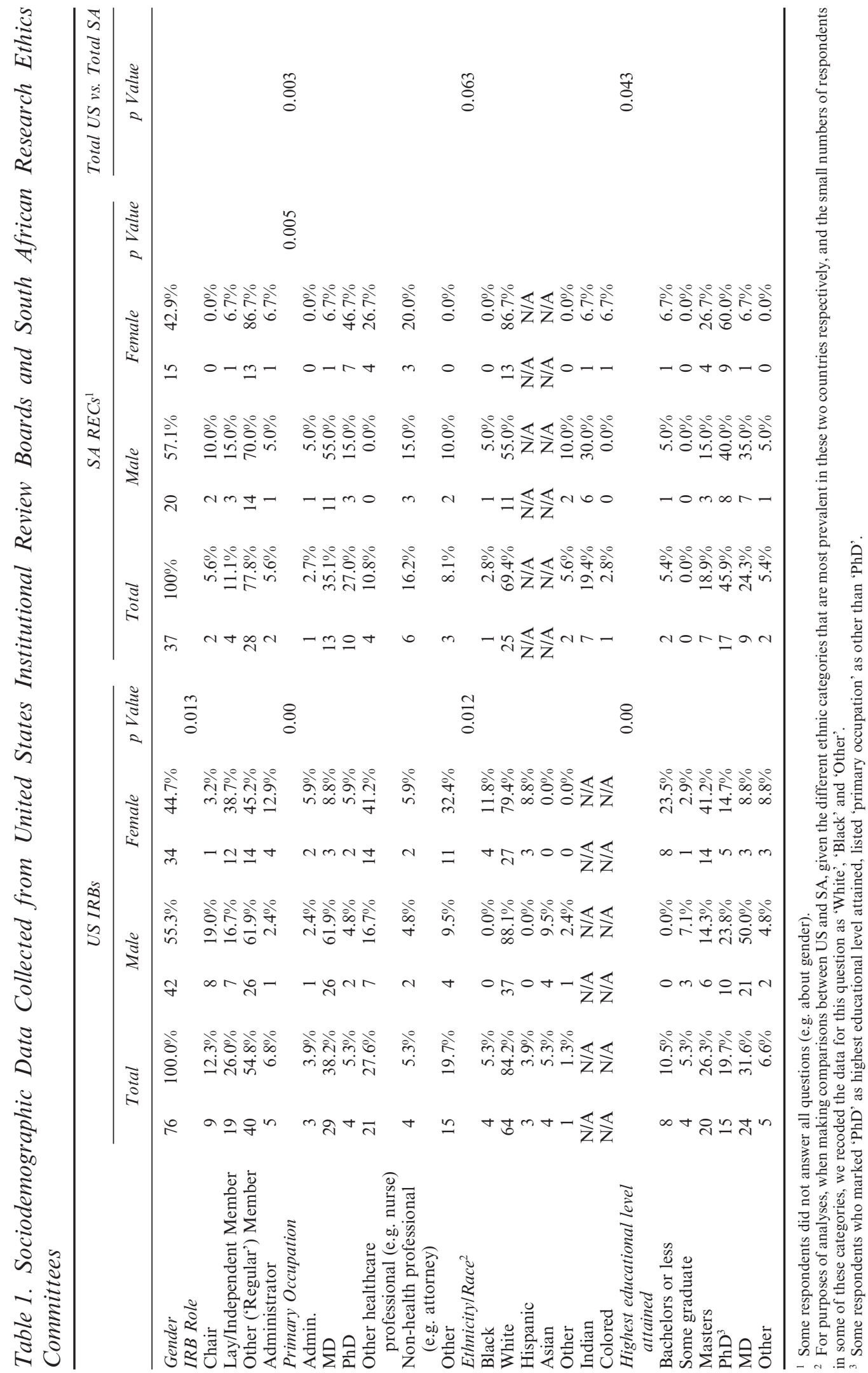


Table 2. Other Data Collected from United States Institutional Review Boards and South African Research Ethics Committee Members

$\frac{\text { US IRB }}{\text { Total (\%) }} \frac{\text { SA REC }}{\text { Total (\%) }} \frac{\text { US vs. SA }}{p \text { Value }}$

\section{PROCESS OF HVT REVIEWS}

Has your IRB reviewed HVTs in the US?

Yes

No

Has your IRB/REC reviewed HVTs in (the developing world/SA)?

Yes

No

Has your IRB/REC called for major changes in HVT protocols?*

Very often

Sometimes

Rarely

Never

Have IRB/REC members disagreed concerning HVT protocols?*

Very often

Sometimes

Rarely

Never

Have you ever approved oral consent for an HVT protocol?**

Yes

No

CONTENT OF HVT REVIEWS

Do you think HVT participants in the developing world/SA understand the risks \& benefits involved in HVTs?**

Very well

Somewhat well

Somewhat poorly

Poorly

Do participants in the developing world/SA understand that they may be given a placebo?**

Very well

Somewhat well

Somewhat poorly

Poorly

Minimum standard of treatment for participants who become HIV-infected during an $H V T$ in the developing world $I S A$

Best avail worldwide

Best avail in host country

Standard in host country

How adequately is the privacy of research participants in HVTs protected in the developing world?**

Very adequately

Somewhat adequately

Somewhat inadequately

Very inadequately

Do you think your IRB/REC would benefit from additional education concerning HIV vaccine trials?

Yes

No

$\begin{array}{rrrr}52 & 69.3 \% & \text { N/A } & \text { N/A } \\ 23 & 30.7 \% & \text { N/A } & \text { N/A } \\ 30 & 41.1 \% & 27 & 77.1 \% \\ 43 & 58.9 \% & 8 & 22.9 \% \\ & & & \\ 2 & 4.1 \% & 6 & 25.0 \% \\ 26 & 53.1 \% & 16 & 66.7 \% \\ 17 & 34.7 \% & 2 & 8.3 \% \\ 4 & 8.2 \% & 0 & 0.0 \% \\ & & & \\ 3 & 6.0 \% & 3 & 12.5 \% \\ 29 & 58.0 \% & 15 & 62.5 \% \\ 13 & 26.0 \% & 6 & 25.0 \% \\ 5 & 10.0 \% & 0 & 0.0 \% \\ & & & \\ 5 & 10.6 \% & 2 & 7.4 \% \\ 42 & 89.4 \% & 25 & 92.6 \%\end{array}$

ISSUES CONCERNING GENERAL IRB PROCESS

Do you think research participants adequately understand informed consent forms?

Very well

Somewhat well

Somewhat poorly

Poorly

Does your IRB communicate with IRBS/RECs in other countries adequately?

Always

Sometimes

Rarely

Never

$\begin{array}{rrrr}1 & 3.8 \% & 6 & 23.1 \% \\ 13 & 50.0 \% & 5 & 19.2 \% \\ 11 & 42.3 \% & 15 & 57.7 \% \\ 1 & 3.8 \% & 0 & 0.0 \%\end{array}$

0.033

$\begin{array}{rrrr}1 & 3.8 \% & 5 & 19.2 \% \\ 13 & 50.0 \% & 10 & 38.5 \% \\ 10 & 38.5 \% & 11 & 42.3 \% \\ 2 & 7.7 \% & 0 & 0.0 \%\end{array}$

$\begin{array}{rrrr}15 & 55.6 \% & 17 & 63.0 \% \\ 4 & 14.8 \% & 3 & 11.1 \% \\ 8 & 29.6 \% & 7 & 25.9 \%\end{array}$

$\begin{array}{rrrr}8 & 32.0 \% & 13 & 52.0 \% \\ 14 & 56.0 \% & 11 & 44.0 \% \\ 3 & 12.0 \% & 1 & 4.0 \% \\ 0 & 0.0 \% & 0 & 0.0 \% \\ & & & \\ 40 & 65.6 \% & 25 & 75.8 \% \\ 21 & 34.4 \% & 8 & 24.2 \%\end{array}$

$\begin{array}{rr}1 & 1.4 \% \\ 50 & 69.4 \% \\ 16 & 22.2 \% \\ 5 & 6.9 \% \\ & \\ 6 & 13.0 \% \\ 18 & 39.1 \% \\ 17 & 37.0 \% \\ 5 & 10.9 \%\end{array}$

0.000 
Table 2. Continued

\begin{tabular}{|c|c|c|c|c|c|}
\hline & \multicolumn{2}{|c|}{$U S I R B$} & \multicolumn{2}{|c|}{$S A R E C$} & \multirow{2}{*}{$\frac{U S \text { vs. } S A}{p \text { Value }}$} \\
\hline & Total & $(\%)$ & Total & $(\%)$ & \\
\hline \multicolumn{6}{|c|}{ Should protocols be reviewed at a centralized, national level rather than locally? } \\
\hline Always & 1 & $1.4 \%$ & 3 & $8.6 \%$ & \\
\hline Sometimes & 30 & $43.5 \%$ & 13 & $37.1 \%$ & \\
\hline Rarely & 28 & $40.6 \%$ & 12 & $34.3 \%$ & \\
\hline Never & 10 & $14.5 \%$ & 7 & $20.0 \%$ & \\
\hline \multicolumn{6}{|c|}{$\begin{array}{l}\text { Do the ethical principles used by IRBs in the US need to be amended by } \\
\text { IRBSIRECS in the developing worldISA? }\end{array}$} \\
\hline Very much & 4 & $7.7 \%$ & 7 & $23.3 \%$ & \\
\hline Somewhat & 24 & $46.2 \%$ & 14 & $46.7 \%$ & \\
\hline Rarely & 15 & $28.8 \%$ & 6 & $20.0 \%$ & \\
\hline Never & 9 & $17.3 \%$ & 3 & $10.0 \%$ & \\
\hline \multicolumn{6}{|c|}{ Do researchers understand and appreciate the role of the IRB/REC? } \\
\hline Very much & 12 & $16.2 \%$ & 5 & $13.9 \%$ & \\
\hline Somewhat well & 60 & $81.1 \%$ & 28 & $77.8 \%$ & \\
\hline Somewhat poorly & 2 & $2.7 \%$ & 3 & $8.3 \%$ & \\
\hline Poorly & 0 & $0.0 \%$ & 0 & $0.0 \%$ & \\
\hline
\end{tabular}

* Indicates answers recorded only for those subjects who had reviewed HVTs in US or SA.

** Indicates answers recorded only for subjects who had reviewed HVTs for a developing country/SA.

Table 3. Questions Collected from South African Research Ethics Committees but not United States' Institutional Review Boards

Total

Male

Female

p Value

How would you describe your level of expertise in the field of ethics?

Basic

Intermediate

Advanced

$\begin{array}{llllll}11 & 30.6 \% & 8 & 40.0 \% & 2 & 13.3 \%\end{array}$

What does your ethics training comprise of?

Self-taught

Attendance of short courses/seminars

Diploma level

Postgraduate level

$\begin{array}{rr}16 & 44.4 \% \\ 9 & 25.0 \% \\ & \\ 13 & 40.0 \% \\ 15 & 42.9 \% \\ 1 & 2.9 \% \\ 5 & 14.3 \%\end{array}$

of the process or content of HVT reviews or the process of IRBs. We considered doing multivariate modeling: however, the numbers of respondents appeared to be relatively low for this purpose.

\section{CONCLUSIONS}

This study is one of the first to offer data on US IRB decision-making, and the first that we know of to compare IRBs/RECs in the US and the developing world. Critical similarities and differences between IRB/REC members in the two countries emerged. The existence of these similarities in the composition and views of members across countries is noteworthy and potentially reassuring to those who are con- cerned about discrepancies between IRBs and the trustworthiness of IRBs/RECs outside the US.

Critical differences between countries arose concerning perceived misunderstandings by HVT participants. Respondents in the US, more than in SA, thought that research participants understood risks and benefits involved in HVTs and consent forms in general. These differences may emerge because SA respondents have more 'on the ground' experience with conducting research in the developing world and are thus more aware of problems in comprehension of key issues, while, from greater distances, US IRBs' assume that participants understand IRBapproved forms. Yet this discrepancy is of concern. Studies have demonstrated relatively high rates of participant misunderstanding of informed consent 
in the West $^{38}$ and in the developing world. ${ }^{39}$ The present data further highlight another aspect of this misunderstanding: that US IRB members assume participants comprehend studies when that may not be the case. US IRB beliefs in the effectiveness of the informed consent process pose potential problems, as these beliefs may undermine effective efforts to obtain truly informed consent, which is ethically necessary. Potentially, in the debate about centralized versus localized IRBs, findings such as these could more fully support the existence of local IRBs that presumably would best be able to assess such details concerning participant comprehension. These findings also pose broader questions about the optimal ability of US IRBs to make decisions that might be made more knowledgeably locally, in the developing world. Other reports have suggested that US IRBS are seen as being overly concerned with consent forms, rather than participant comprehension per se. ${ }^{40}$ The present data highlight another aspect of this phenomenon that may partly underlie it: IRB members' belief that participants in fact understand these forms. These data also highlight the need for IRBs in the developing world and the US to communicate better, given differences in views of this communication and in perceptions of HVT participant comprehension.

The fact that, in both countries, most members thought they needed additional training, despite educational efforts over the past few years, indicates the importance of establishing and maintaining appropriate educational initiatives. The finding that $40 \%$ of SA members reported being self-taught is also of concern. Future research can explore whether those who are 'self-taught' view or approach ethical issues differently than others (i.e. those who have had more formal ethics training) and, if so, how; of what self-teaching consists (e.g. the range, quantity, and content of self-education in ethics); how widespread it is (i.e. in the US and other countries); how it may be limited, and can best be complemented or enhanced (i.e. in what specific areas further training is required).

\footnotetext{
${ }^{38}$ S. Joffe et al. Quality of Informed Consent in Cancer Clinical Trials: A Cross-sectional Survey. Lancet 2001; 358: 1772-1777.

${ }^{39}$ M. Krosin et al. Problems in Comprehension of Informed Consent in Rural and Peri-urban Mali, West Africa. Clin Trials J 2006; 3: 306-313.

${ }^{40}$ Sugarman et al., op. cit. note 25 .
}

In both countries, members were divided on several issues: for example, on the minimum standard for treatment for participants who become HIV-infected during HVTs. While, a priori, one might have expected SA members to be more likely to favor the highest possible standard (i.e. wanting the most for their comparatively resource-poor country), $37.1 \%$ of members in SA and $44.4 \%$ of members in the US thought that other lesser levels of treatment were appropriate and responses from the two countries did not significantly differ. Hence, a sizable proportion of members appeared to be pragmatic in their approach - choosing not the highest, but a more feasible standard - rather than adhering rigidly to universalistic principles that treatment should not vary at all between countries.

In both countries, respondents were also almost equally divided on needs for local versus 'centralized national level' review. Future research can investigate what factors separate those who think that central rather than local review is appropriate and on what protocols members think central review would be advantageous. Prior discussions have focused on whether central ( $v s$. local) review is better rather than on what specific types of protocols (other than multi-site studies in general) would be best for each approach. ${ }^{41}$ The fact that respondents themselves are divided also needs to be taken into consideration by policy-makers considering whether to alter the current system of local review. Clearly, policy-makers need to consider not only whether such a central system would, in the abstract, ensure more optimal reviews, but how such a system would be instituted, given that IRB/ REC members are divided about it, with many opposing it.

In both countries, respondents were also split as to whether principles need to be 'amended' in the developing world. As mentioned earlier, ethical relativism has received attention, ${ }^{42}$ with commentators

\footnotetext{
${ }^{41}$ J. Gold \& C.S. Dewa. Institutional Review Boards and Multisite Studies in Health Services Research: Is there a Better Way? Health Serv Res 2005; 40: 291-307; R.D. Pentz \& A.F. Khayat. The Poster Child for the Need for Central Review of Research Protocols: The Children's Oncology Group. Camb Q Healthc Ethics 2004; 13: 359-365.

${ }^{42}$ Angell, op. cit. note 2; Lurie \& Wolfe, op. cit. note 2; Beauchamp, op. cit. note 15; Macklin, op. cit. note 16; Christakis, op. cit. note 17; Turner, op. cit. note 18; Barry, op. cit. note 18.
} 
on each side. Yet the present data suggest that resolution of this issue may be much more complicated than some observers may anticipate and that more than simple binary approaches toward relativism (i.e. of pro and con) may be needed. Rather, challenges emerge of developing appropriately nuanced understandings of underlying issues and assumptions, exploring factors involved and integrating these considerations and complexities into professional training. Future research can investigate when, how and why some members think principles need to be amended.

Of note, in both countries, the majority of members were white. In SA, the low numbers of blacks on committees was particularly striking. Future research can explore the fact that gender was associated with professional training and, in the US, with IRB role. Gender, though not significantly associated here with differences in views of the content or process of reviews, may be related to other aspects of these areas that were not probed here (e.g. in studies that explicitly involve gender roles, such as research concerning reproductive issues).

The problem of achieving ethnic diversity in membership of IRBs/RECs appears not to be unique to one country and may reflect and contribute to tensions more generally between medical researchers and lay communities. Of note, subsequent in-depth semi-structured interviews that I have been conducting in SA with REC members, following the administration of this questionnaire (and on which I plan to report separately), have indicated that these members view this unequal ethnic distribution as a problem and are trying to resolve it but face several critical obstacles. Community members may lack the education to understand fully complicated scientific protocols under review. Community members who have achieved sufficient education, and are thus health care professionals themselves, may no longer at that point truly be 'community members' but rather members of the research enterprise, broadly defined. RECs require long hours and dedication, and community members often face impediments of competing time pressures. Hence, REC members report that community members often 'drop out' of RECs after only a few months or a year. These problems could potentially be addressed not just by individual institutions, but at larger levels as well (e.g. through WHO or UNESCO). Specifically, needs exist to develop programs and incentives to engage and educate community members broadly about research and RECs.

This study has several potential limitations. The sample size is comparatively small, but still large enough to illustrate and highlight key issues that require further attention by members of the research enterprise (e.g. IRB/REC members and policymakers) and by researchers in ethics, and others. Though the response rates are not as high as some studies of other populations, IRB/REC members are a particularly difficult group to study because, as described above, their institutions do not ordinarily reveal their names. As mentioned, these logistical obstacles to studying IRB/REC members have led to relatively few studies being conducted to date. The response rates here are also reasonable for studies of busy health care professionals. ${ }^{43}$ In comparison, some other mail surveys track whether individual survey recipients have responded yet and, if not, re-contact them and mail surveys three times. The fact that IRBs will not reveal membership to researchers, and the need to maintain anonymity of respondents, hampered possibilities of re-contacting recipients individually. A structured questionnaire was used, which did not allow participants to elaborate responses to questions and terms (e.g. how exactly ethical principles might 'need to be amended in the developing world/SA'). However, these domains can be investigated further in future research. Despite these limitations, these data are very valuable, offering initial exploratory data in this area. Though SA is not representative of all developing countries, as a sub-Saharan African nation in the post-apartheid era, it shares many challenges in terms of epidemics of HIV and other

\footnotetext{
${ }^{43}$ R. Klitzman, A. Bodkin \& H.G. Pope. Sexual Orientation and Associated Characteristics Among North American Academic Psychiatrists. J Sex Res 1998; 35: 282-287; A. Bodkin, R. Klitzman \& H. Pope. Treatment Orientation and Associated Characteristics Among North American Academic Psychiatrists. J Nerv Ment Dis 1995; 184: 729-735; R.R. Acton et al. Knowledge, Attitudes, and Behaviors of Alabama's Primary Care Physicians Regarding Cancer Genetics. Acad Med 2000; 75: 850-852; E. Mountcastle-Shah \& N.A. Holtzman. Primary Care Physicians' Perceptions of Barriers to Genetic Testing and their Willingness to Participate in Research. Am J Med Genet 2000; 94: 409-416.
} 
infectious diseases, low levels of education and literacy, post-colonial political problems, and limited resources. Further research can examine these issues in other countries as well. As noted, for this pilot study we did not gather data on names of institutions or IRB/RECs (given that some institutions have more than one IRB) in order to ensure maximal protection of confidentiality. However, all of the chairs to whom questionnaires had been sent had first agreed to distribute these surveys to their members. Hence, it is reasonable to assume that at least some members from each committee completed and returned the questionnaire. Future research can explore differences between views of members of any particular IRB/REC, and compare individual IRB/RECs. Due to the intent to keep the questionnaire as short as possible, to reduce the burden on busy professionals, we were not able to probe certain areas in further depth. Hence, we left certain terms (e.g. about how often IRBs request 'major changes', and 'disagree', etc.) to be defined by participants themselves. Though some questions may be vague, the data suggest similarities and differences in IRB/REC members' views and practices concerning these domains that future research can investigate further. Thus, though these potential limitations may possibly lower the generalizability of the study, this research nevertheless provides important suggestive data. These exploratory data can provide vital background for future larger studies that can pursue these areas further among larger numbers of committees, using surveys that request names of IRBs and institutions as well.

Another potential limitation is the possible effect of multiple comparisons. The significance levels presented are not corrected for the effects of multiple comparisons. Chance associations may have occurred due to the number of comparisons, particularly when we assessed comparisons of marginal statistical significance. However, classical Bonferroni corrections tend to be overly conservative in research such as this. In addition, a consistent overall pattern emerged broadly across variables and these findings appear to have a certain face validity. We present the findings here without correction, allowing readers to judge the results for themselves. As mentioned, the findings can be pursued more thoroughly in future studies as well.

As suggested above, given how comparatively few studies have been conducted on IRBs, despite these limitations, these descriptive findings are thus important. Surveys were here distributed to chairs, and because these questionnaires focused on one issue - HVTs, which have raised wide public discussion - IRB/REC chairs and members appeared to recognize the value of the project and consequently may have been amenable to participating. Future researchers may want to use similar approaches - making studies of IRBs 'issue specific'. However, these data also reveal another obstacle to research of which future studies will need to take account: that IRBs/RECs may be hard to study about a particular issue, in part because members regularly 'rotate off'.

In sum, these data shed important initial light on several crucial areas concerning the process and content of IRB/REC reviews of HVTs in the developing and the developed world. Given rapidly increasing globalization and collaboration between developed and developing nations in investigating a variety of disorders, this study thus has key implications for future research and scholarship (in both ethics and prevention of HIV and other disorders), policy, and practice concerning the ethical review of a widening range of study protocols in upcoming years.

\footnotetext{
Acknowledgements

The author would like to thank Jeff Severson, Scott Reiners, Davin Skalinder, Lauren Stelljes, Jerome Singh and Mary DuVernay for their assistance with the preparation of this manuscript.
} 